\title{
Warm-Core Eddies Studied by Laboratory Experiments and Numerical Modeling
}

\author{
Angelo Rubino \\ Institut für Meereskunde, Universität Hamburg, Hamburg, Germany \\ PETER BRANDT \\ Institut für Meereskunde an der Universität Kiel, Kiel, Germany
}

(Manuscript received 21 December 2001, in final form 19 August 2002)

\begin{abstract}
Aspects of the dynamics of warm-core eddies evolving in a deep ocean are investigated using the results of laboratory experiments and numerical simulations. The vortices, produced experimentally in a system brought to solid body rotation by rapidly lifting a bottomless cylinder containing freshwater immersed in a salty ambient fluid, show clearly the presence of inertial oscillations: deepenings and contractions, shoalings and expansions, alternate during an exact inertial period. These pulsations, though predicted analytically and simulated numerically, had never been measured before for surface eddies having aspect ratios, as well as Rossby and Burger numbers, typical of geophysical warm-core eddies. The spatial structure of the vortex radial and tangential velocity components is analyzed using the experimental results and numerical simulations carried out by means of a layered, nonlinear, reduced-gravity frontal model. It is found that, while the dependence of the vortex radial velocity on the vortex radius evolves toward linearity as time elapses, different spatial structures seem to be possible for the vortex tangential velocity dependence. This behavior, which strongly differs from the "pulson" dynamics, is instead consistent with recently found analytical solutions of the nonlinear, reduced-gravity shallowwater equations describing the dynamics of warm-core eddies on an $f$ plane.
\end{abstract}

\section{Introduction}

A great deal of attention has been paid in the past three decades to the study of warm-core eddies (see, e.g., Saunders 1971; Olson et al. 1985; Rogers 1989; Matsuura 1995; Cushman-Roisin and Merchant-Both 1995, Rubino et al. 1998; 2002). These features of the oceanic mesoscale, consisting of anticyclonically rotating isolated water masses of anomalous water, are considered in fact to play an important role in the larger scale ocean circulation as, for example, they can profoundly affect the transfer of physical, chemical, and biological properties across frontal zones and exert a substantial contribution to the horizontal and vertical mixing of different waters masses (Olson 1991). In particular, this last phenomenon seems to be also associated with the capacity of mesoscale frontal features in general and warm-core eddies specifically of transforming long, mostly wind-generated, near-inertial waves into shorter ones, which are able to rapidly propagate their energy toward the abyss (see, e.g., Kunze 1985, 1986; Klein and Treguier 1993; Lee and Eriksen 1997; Chant 2001).

Corresponding author address: Angelo Rubino, Institut für Meereskunde, Universität Hamburg, Troplowitzstr. 7, D-22529 Hamburg, Germany.

E-mail: rubino@ifm.uni-hamburg.de
On the other hand, it is known theoretically that warmcore eddies possess intrinsic modes of near-inertial oscillations (Cushman-Roisin 1987; Rogers 1989; Rubino et al. 1998). Accordingly, in the theoretical study of warm-core eddies two large classes of investigations can be identified. The first class is composed of investigations aimed at studying aspects of the interactions between these eddies and their environment. In particular, studies that belong to this class deal with vortex generation by geostrophic adjustment (Csanady 1979); meandering of boundary currents (Saunders 1971; Evans et al. 1984), energy radiation via Rossby wave (Chassignet and Cushman-Roisin 1991) or gravity wave emission (Ungarish et al. 2001); interaction with the nearinertial ambient wave field (Kunze 1985, 1986; Kunze et al. 1995); decay by interfacial friction, diffusion, and ambient water entrainment (Csanady 1979; Rubino et al. 2002); as well as vortex-vortex (Pavia and CushmanRoisin 1990) or vortex-current interactions (CushmanRoisin and Merchant-Both 1995). On the other hand, studies focusing on intrinsic characteristics of warmcore eddies, such as those based on analytical and numerical models describing aspects of their dynamics, constitute the elements of the second class.

In particular, in the frame of the nonlinear, reducedgravity, shallow-water equations on an $f$ plane, analyt- 

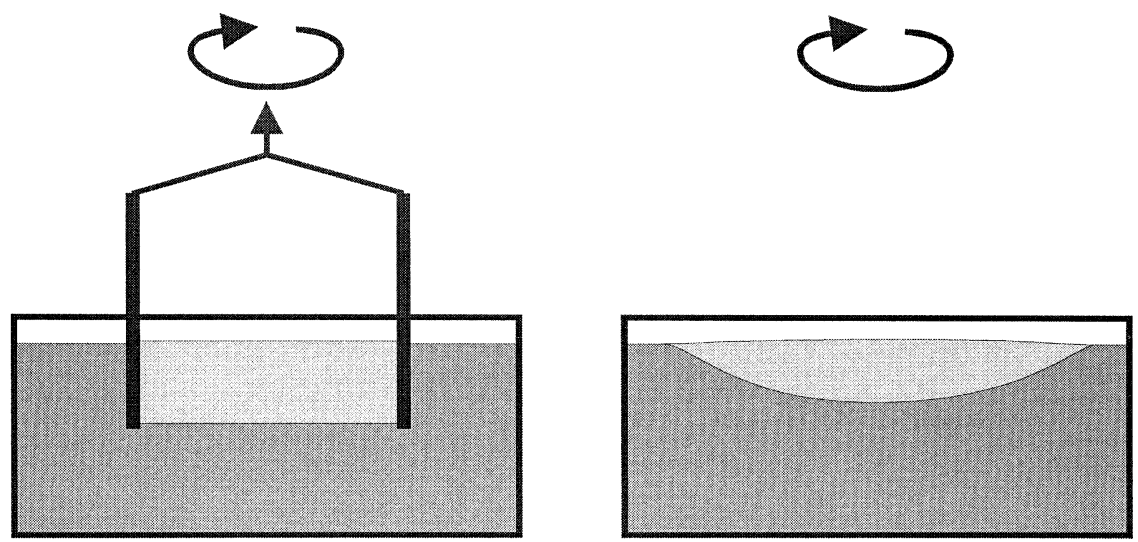

FIG. 1. Schematic representation of the apparatus used to generate warm-core eddies in the rotating tank.

ical solutions describing paraboloidic warm-core eddies characterized by elliptical horizontal sections were found by Cushman-Roisin (1987) and Rogers (1989). These solutions exhibit, among other things, two intriguing peculiarities: The vortex horizontal velocity components are linear functions of the horizontal coordinates, and they pulsate with exact inertial frequency (hence the name "pulson" with which they were baptized). An extension of these solutions to circular vortices with more general tangential velocity profiles and shapes was presented by Rubino et al. (1998). However, even in this case, the radial velocity dependence on the horizontal coordinates is linear and the vortex still pulsates inertially. Thus, these two characteristics seem to be intrinsically associated to the dynamics of circular warm-core eddies evolving on an $f$ plane. In this context, the robustness of the inertial oscillation has been recently demonstrated in the frame of a theoretical study carried out numerically by Rubino et al. (2002) for circular warm-core eddies of the nonlinear, reduced-gravity, shallow-water equations evolving under the effect of linear, as well as quadratic, interfacial friction, harmonic horizontal eddy diffusion, and linear ambient water entrainment. However, this oscillatory behavior as well as the linear dependence of the vortex radial velocity on the vortex radius has never been observed either in the ocean or in laboratory.

In this paper we present results from laboratory experiments describing the dynamics of warm-core eddies having aspect ratios typical of geophysical vortices. The produced vortices show clearly the presence of inertial oscillations. The spatial structure of the vortex radial and tangential velocity components is analyzed using the experimental results as well as numerical simulations carried out by means of a nonlinear, reduced-gravity frontal model. It is found that, while the dependence of the radial velocity on the vortex radius evolves toward linearity as time elapses, different spatial structures seem to be possible in the case of the tangential velocity dependence.

\section{Methodology}

The results of this investigation are both experimental and numerical. The experimental results were obtained using the rotating tank of LEGI/Coriolis (Grenoble, France), which, owing to its 13-m diameter, allows for the investigation of vortices having aspect ratios as well as Rossby and Burger numbers typical of geophysical warm-core eddies. The vortices were produced in a system brought to solid body rotation by rapidly lifting a 2-m-radius bottomless cylinder containing freshwater immersed in a salty ambient fluid (Fig. 1). Time series of interface displacement were measured using a set of interface followers located at different distances from the vortex center. These probes measure the time interval that an acoustic signal needs to vertically propagate from a source above an interface between two water layers to a reflector below this interface and back. As the temperature is virtually constant in our experiments, this time is a function of the salinity difference between both layers. Thus by using these probes the movement of the interface separating two fluids of different salinity can be measured accurately (Renouard et al. 1993; Ramirez and Renouard 1998). Moreover, time series of the vortex radial and tangential velocity were measured using ultrasonic Doppler probes that have a precision of about $0.1 \mathrm{~mm} \mathrm{~s}^{-1}$ for velocities in the range $0-20 \mathrm{~cm}$ $\mathrm{s}^{-1}$.

The numerical results were obtained, for the same experimental initial conditions characterizing the vortex structure, using the layered model described in detail in Rubino et al. (2002). This model solves the nonlinear shallow-water equations in the frame of the reducedgravity assumption. Because of a numerical technique for the treatment of movable lateral boundaries, the model is capable of simulating expansions and contractions of the vortex surface area. The model was preliminarily run including dissipation terms; that is, quadratic interfacial friction and harmonic horizontal eddy viscosity were considered. Other dissipative mech- 

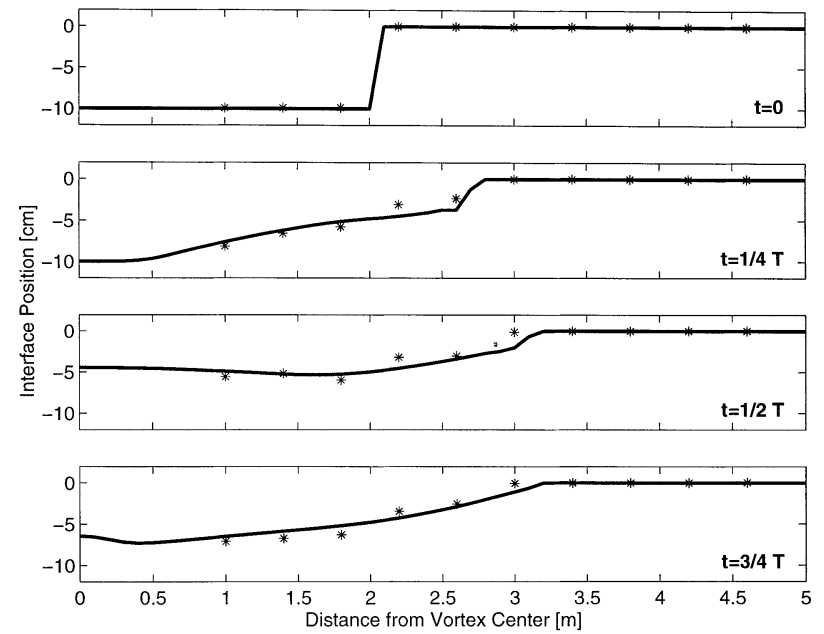

FIG. 2. Interface depth at the initial stage of the experiment as well as at three phases after the lift of the bottomless cylinder (stars). Also included are the simulated curves.

anisms in this model are those inherent in the adopted numerical schemes. They depend on the chosen temporal and spatial resolution. In our numerical simulations we used a time step of $2 \mathrm{~s}$ and a grid size of 0.1 $\mathrm{m}$. With this resolution best agreement between model and experimental results were obtained for vanishing values of interfacial friction and eddy viscosity coefficients. A detailed discussion on the influence of linear and quadratic interfacial friction as well as harmonic eddy viscosity and linear water entrainment on the decay of stable warm-core eddies can be found in Rubino et al. (2002). In the next section, experimental results and numerical simulations will be used to analyze the evolution of the structure of the simulated vortex displacement and velocity field.
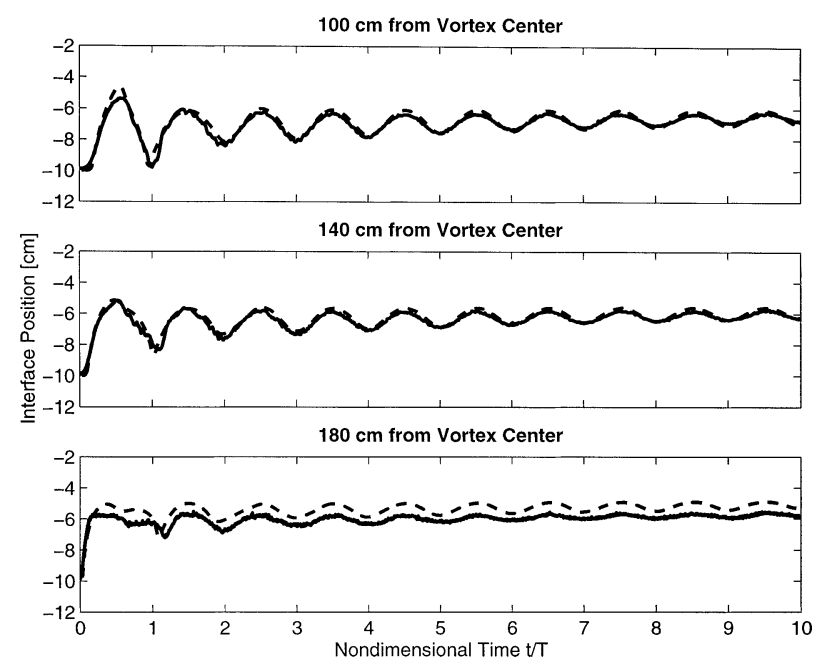

FIG. 3. Comparison between interface positions as obtained experimentally (solid lines) and as simulated numerically (dashed lines).

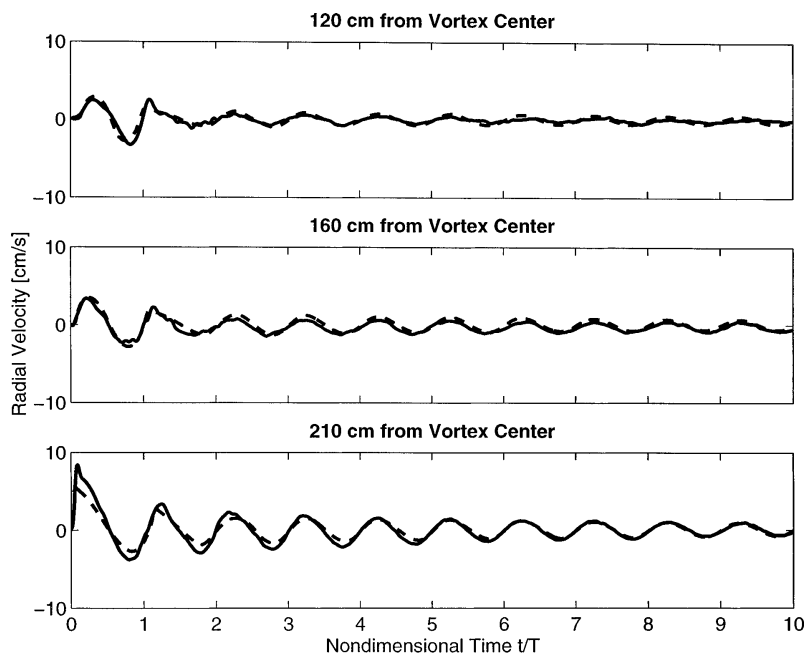

FIG. 4. Comparison between radial velocities as obtained experimentally (solid lines) and as simulated numerically (dashed lines).

\section{Results}

The parameters characterizing the experiment to which we will refer in the present section are relative density difference $\Delta \rho / \rho=0.925 \times 10^{-3}$, initial eddy thickness $H=10 \mathrm{~cm}$, initial eddy radius $L=2 \mathrm{~m}$, total water depth $D=86 \mathrm{~cm}$, and rotation period of the tank $T=120 \mathrm{~s}$. Note that, as discussed in Rubino et al. (2002), for this kind of parameters the reduced-gravity assumption is valid for the description of the two-layer vortex evolution during at least the first 10 inertial periods. Given the above mentioned vortex parameters, the vortex Burger number is

$$
\mathrm{Bu}=\frac{g \Delta \rho / \rho H}{f^{2} L^{2}}=0.02,
$$

which belongs to the range of Burger numbers typical for warm-core eddies in the ocean. In Fig. 2 the interface depth at the initial stage of the experiment as well as at three successive phases after the lift of the bottomless cylinder as obtained experimentally and as simulated numerically are depicted. After the cylinder is lifted, a strong vortex expansion occurs (Figs. 2b, and 2c), characterized by a strong increase of the vortex radius and a strong decrease of the vortex thickness. This phase is followed by a remarkable vortex contraction (Fig. 2d) connected with a deepening of the vortex core (note that, especially in the numerical results which are unavoidably affected by numerical dissipation particularly near the vortex rim, a less pronounced decrease in the vortex radius is obtained). Time series of the vortex interfacial position at 100,140 , and $180 \mathrm{~cm}$ from the vortex center, of the vortex radial velocity at 120 and $160 \mathrm{~cm}$ from the vortex center, and of the vortex tangential velocity at 120,160 , and $210 \mathrm{~cm}$ from the vortex center as obtained experimentally and as calculated numerically are depicted in Figs. 3, 4, and 5, respectively. 


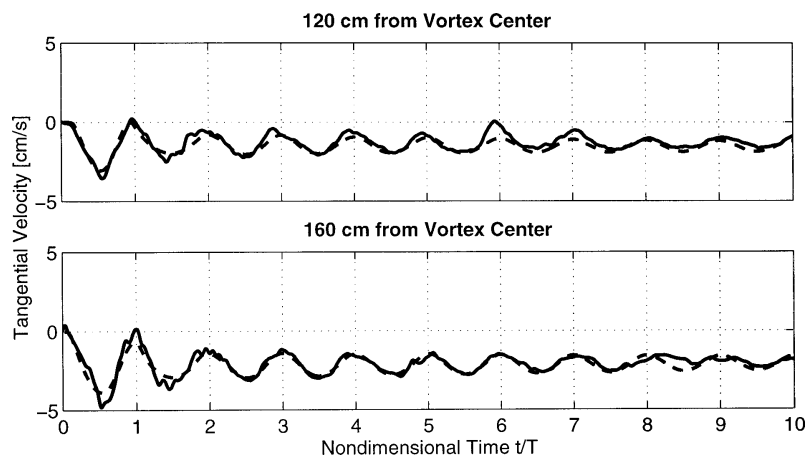

FIG. 5. Comparison between tangential velocities as obtained experimentally (solid lines) and as simulated numerically (dashed lines).

Note that the vortex Rossby number Ro $=U / f L$, which results using a mean tangential velocity of $U=2 \mathrm{~cm}$ $\mathrm{s}^{-1}$, is about 0.1 . This value belongs to the range of Rossby numbers typical for warm-core eddies in the ocean.

In general, good agreement between experimental results and model results is observed. However, noticeable discrepancies between measured and simulated interface positions (see, e.g., Figs. 3a and 3b) exist. They can be particularly ascribed to the uncertainty related to the experimental determination of the vortex interface in the presence of mixing, which does not arise in the numerical simulation. Both measured and simulated behavior shows clearly the presence of inertial oscillations: vortex expansions, associated with outward radial velocity and enhanced tangential velocity, and vortex contractions, associated with inward radial velocity and reduced tangential velocity, alternate during an exact inertial period. This evolution is consistent with known analytical solutions describing the dynamics of warmcore eddies of the nonlinear, reduced-gravity, shallowwater equations evolving on an $f$ plane (Cushman-Roisin 1987; Rogers 1989).

The temporal evolution of the spatial structure of the vortex radial velocities measured in the three locations given in Fig. 4 is assessed using a linear fit. In Fig. 6 the slope of the calculated fit (Fig. 6a) and the norm of the obtained residuals (Fig. 6b) are presented. While inertial oscillations are found again (after an initial adjustment phase) in the alternating sign of the slope, the norm of the residuals rapidly becomes small, suggesting that a rapid evolution of the spatial structure of the vortex radial velocity toward linearity occurs. This behavior may correspond either to a damping of radially propagating internal gravity waves or to its transformation, at the vortex rim, into surface gravity waves radiating energy away from the vortex. The results obtained experimentally are consistent with the results of our numerical simulations (Fig. 7): The norms of the residuals of the simulated vortex radial velocity becomes, in fact, small as time elapses (Fig. 7a). However, the evolution of the structure of the simulated vortex
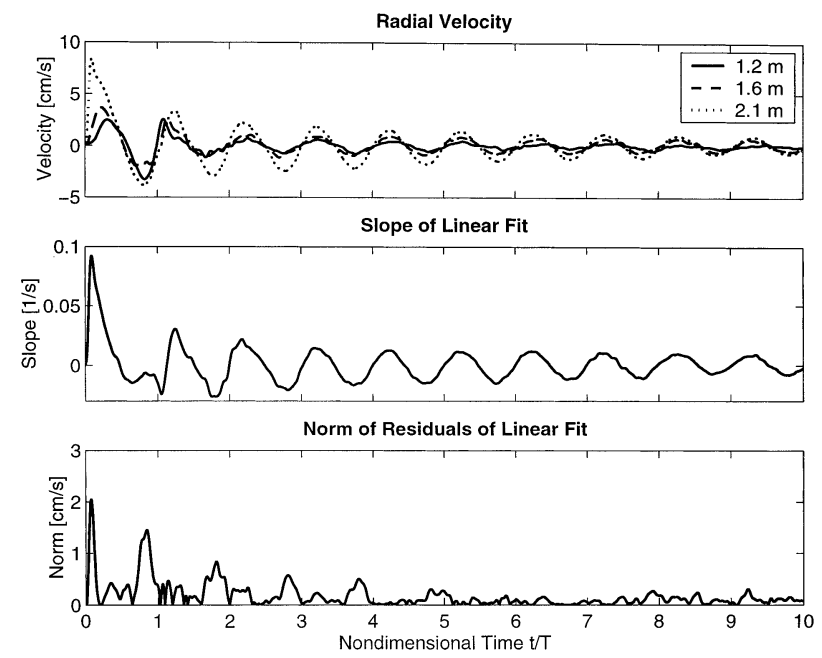

FIG. 6. Time series (top) of the radial velocity at the three positions given in Fig. 4, (middle) of the slope of the linear fit to the spatial structure of the radial velocity, and (bottom) of the norm of the residuals of the linear fit.

tangential velocity is quite different: From its norm it can be evinced that the spatial structure of this velocity component rapidly attains a quasi-periodic state that is far from linearity (Fig. 7a). It also significantly deviates from quadraticity (Fig. 7b), while a much better agreement with a cubic structure is found (Fig. 7c). This deviation from linearity means that the vortex rotation deviates from solid-body rotation and, hence, that the vortex relative vorticity is in general not constant within the vortex core.

\section{Conclusions}

In this investigation, for the first time, experimental results addressing aspects of the unsteady, pulsating dy-
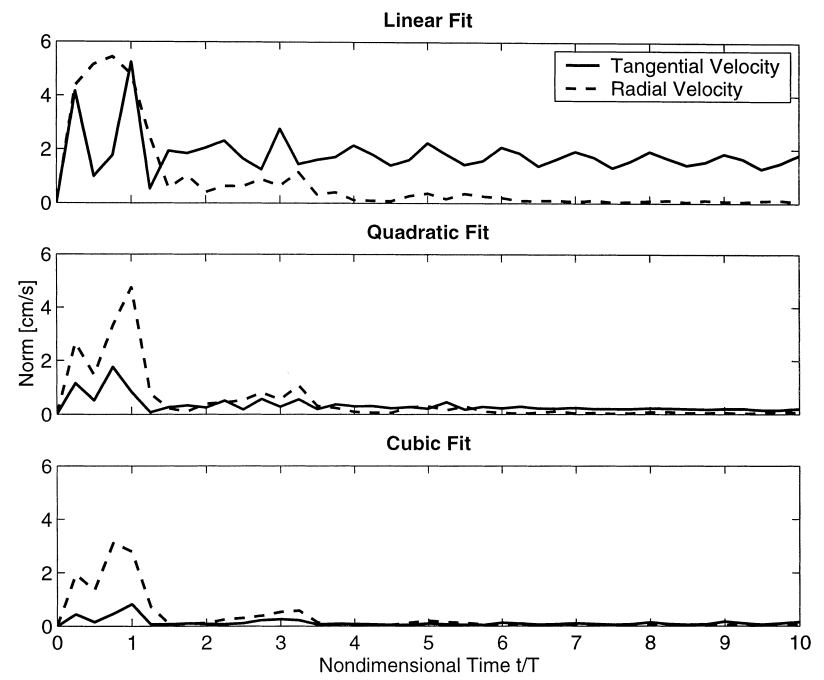

FIG. 7. Simulated norm of residuals to the (top) linear, (middle) quadratic, and (bottom) cubic fit of radial (dashed lines) and tangential (solid lines) velocity. 
namics of warm-core eddies having aspect ratios typical for geophysical vortices were presented. We were able to obtain these measurements because we used the rotating tank of LEGI/Coriolis (Grenoble, France), which, owing to its $13-\mathrm{m}$ diameter, allows for the reproduction of flow features characterized by large Reynolds numbers and small Rossby and Burger numbers. While inertial oscillations and linear spatial structure of the radial velocity emerge as very robust characteristics of the measured vortex dynamics, the behavior of the measured and of the simulated vortex tangential velocity strongly deviates from the behavior that can be inferred from different analytical solutions of the nonlinear, reduced-gravity, shallow-water equations describing the evolution of warm-core eddies on an $f$ plane (CushmanRoisin 1987; Rogers 1989). These solutions are, in fact, characterized by a linear structure of the vortex radial as well as tangential velocity. In recently discovered analytical solutions describing the same kind of vortices in the same context (Rubino et al. 1998) a more general structure of the vortex tangential velocity (and hence a more general vortex shape than the paraboloidic one characterizing the previous solutions) is allowed, while linearity still represents the only possible structure of the vortex radial velocity. Our results indicate that, indeed, vortices characterized by a velocity structure consistent with the solution proposed by Rubino et al. (1998) emerge by the impulsive release of a motionless cylinder of buoyant fluid in a deep environment on an $f$ plane. Numerical and experimental results agree in indicating a strong tendency toward linearity of the spatial structure of the vortex radial velocity. This tendency is connected with the rapid decrease of the energy associated with radially propagating internal gravity waves generated during the initial adjustment phase of the vortex evolution. Their dissipation, in the experimental results as well as in the numerical simulations, cannot be described by an inviscid, reduced-gravity theory. However, the spatial vortex structure found by Rubino et al. (1998) seems to be preserved even in the presence of dissipative mechanisms. This result is consistent with the findings of Rubino et al. (2002), who showed that, in the short-term evolution of a warm-core eddy, linear as well as quadratic interfacial friction, harmonic eddy viscosity, linear water entrainment, as well as an active lower layer, are not able to modify substantially the spatial vortex structure typical of the analytical "pulson" solution described in detail by Cushman-Roisin (1987). Thus, the analytical and numerical predictions, indicating that warm-core eddies exist also far from solid-body rotation (i.e., far from the "pulson" structure), is confirmed by the present laboratory experiments.

Acknowledgments. This study has been partly funded by the European Commission under Contract HPRI-CT-
1999-00006 and by the Deutsche Forschungsgemeinschaft in the frame of the Sonderforschungsbereich 512. We thank Felix Morsdorf, Joël Sommaria, Henri Didelle, René Carcel, Stephane Mercier, and the whole staff of LEGI/Coriolis for their help during the experimental phase of our investigation.

\section{REFERENCES}

Chant, R., 2001: Evolution of near-inertial waves during an upwelling event on the New Jersey inner shelf. J. Phys. Oceanogr., 31, 746-764.

Chassignet, E. P., and B. Cushman-Roisin, 1991: On the influence of a lower layer on the propagation of nonlinear oceanic eddies. $J$. Phys. Oceanogr., 21, 939-957.

Csanady, G. T., 1979: The birth and death of a warm core ring. $J$. Geophys. Res., 84, 777-780.

Cushman-Roisin, B., 1987: Exact analytical solution for elliptical vortices of the shallow-water equations. Tellus,39A, 235-244.

- and S. Merchant-Both, 1995: Elliptical warm-core rings in a two-layer ocean with ambient shear. J. Phys. Oceanogr., 25, 2011-2024.

Evans, R. H., K. S. Baker, O. B. Brown, R. C. Smith, S. Hooker, D. Olson, and the Warm-Core-Rings Program Service Office, 1984: Satellite images of warm-core ring $82-\mathrm{B}$, sea surface temperature and a chronological record of major physical events affecting ring structure. WHOI Rep., 25 pp.

Klein, P., and A. M. Treguier, 1993: Inertial resonance induced by an oceanic jet. J. Phys. Oceanogr., 23, 1897-1915.

Kunze, E., 1985: Near-inertial wave propagation in geostrophic shear. J. Phys. Oceanogr., 15, 544-565.

_ 1986 : The mean and near-inertial velocity fields in a warmcore ring. J. Phys. Oceanogr., 16, 1444-1461.

_- R. W. Schmitt, and J. M. Toole, 1995: The energy balance in a warm-core ring's near-inertial critical layer. J. Phys. Oceanogr., 25, 942-957.

Lee, C. M., and C. C. Eriksen, 1997: Near inertial internal waves interactions with mesoscale fronts: Observations and models. $J$. Geophys. Res., 102, 3237-3253.

Matsuura, T., 1995: The evolution of frontal-geostrophic vortices in a two-layer ocean. J. Phys. Oceanogr., 25, 2298-2318.

Olson, D. B., 1991: Rings in the ocean. Annu. Rev. Earth Planet. Sci., 19, 283-311.

_, R. W. Schmitt, M. Kennelly, and T. M. Joyce, 1985: A twolayer diagnostic model of the long-term physical evolution of warm-core ring 82B. J. Geophys. Res., 90, 8813-8822.

Pavia, E. G., and B. Cushman-Roisin, 1990: Merging of frontal eddies. J. Phys. Oceanogr., 20, 1886-1906.

Ramirez, C., and D. P. Renouard, 1998: Generation of internal waves over a shelf. Dyn. Atmos. Oceans, 28, 107-125.

Renouard, D. P., G. G. Tomasson, and W. K. Melville, 1993: An experimental and numerical study of nonlinear internal waves. Phys. Fluids A, 5, 1401-1411.

Rogers, C., 1989: Elliptic warm-core theory: The pulsrodon. Phys. Lett., 138, 267-273.

Rubino, A., P. Brandt, and K. Hessner, 1998: Analytical solutions for circular eddies of the reduced-gravity, shallow-water equations. J. Phys. Oceanogr., 28, 999-1002.

_, K. Hessner, and P. Brandt, 2002: Decay of stable warm-core eddies in a layered frontal model. J. Phys. Oceanogr., 32, 188201.

Saunders, P. M., 1971: Anticyclonic eddies formed from shoreward meanders of the Gulf Stream. Deep-Sea Res., 18, 1207-1220.

Ungarish, M., M. A. Hallworth, and H. E. Huppert, 2001: Axisymmetric gravity currents in a rotating system: Experimental and numerical investigations. J. Fluid Mech., 447, 1-29. 